\title{
Para-mixed linear spaces
}

\author{
Mircea Crasmareanu \\ Faculty of Mathematics \\ University "Al. I. Cuza", Iaşi, România \\ http://www.math.uaic.ro/ mcrasm \\ email: mcrasm@uaic.ro
}

\begin{abstract}
We consider the paracomplex version of the notion of mixed linear spaces introduced by M. Jurchescu in [4] by replacing the complex unit $i$ with the paracomplex unit $j, j^{2}=1$. The linear algebra of these spaces is studied with a special view towards their morphisms.
\end{abstract}

\section{Introduction}

It is well-known that up to isomorphisms there are three 2-dimensional real algebras: $\mathbb{C}=\mathbb{R}[X] /\left(x^{2}+1\right), \mathbb{A}=\mathbb{R}[X] /\left(x^{2}-1\right), \mathbb{D}=\mathbb{R}[X] /\left(x^{2}\right)$. The theory of the first algebra is richer than the other two, a fact corresponding to the field property of $\mathbb{C}$. Similar to the complex case, the paracomplex algebra $\mathbb{A}$ has the basis $\{1, j\}$ with $j^{2}=1$; therefore the elements of $\mathbb{A}$ are $z=x+j y$ with $x$ and $y$ real numbers. For historical details about the paracomplex algebra please see the survey [3].

Similar to the linear complex geometry there exists a paracomplex version as follows: let $\mathrm{V}$ be a real linear space. A paracomplex structure on $\mathrm{V}$ is an involution $\mathrm{J}: \mathrm{V} \rightarrow \mathrm{V}, \mathrm{J}^{2}=1_{\mathrm{V}}$, such that the eigenspaces $\mathrm{V}_{ \pm}:=\operatorname{ker}\left(1_{\mathrm{V}} \pm \mathrm{J}\right)$ have the same dimension. The pair $(\mathrm{V}, \mathrm{J})$ is then called a paracomplex linear space. If the hypothesis regarding the eigenspaces is dropped then we obtain the notion of almost paracomplex structure. An $\mathbb{A}$-linear map between the (almost) paracomplex linear spaces $(\mathrm{V}, \mathrm{J})$ and $\left(\mathrm{V}^{\prime}, \mathrm{J}^{\prime}\right)$ is a linear map $\mathrm{T}: \mathrm{V} \rightarrow \mathrm{V}^{\prime}$

2010 Mathematics Subject Classification: 15A03, 15A04

Key words and phrases: paracomplex structure, para-mixed linear space, morphism 
satisfying $\mathrm{T} \circ \mathrm{J}=\mathrm{J}^{\prime} \circ \mathrm{T}$. Just like complex vector spaces are vector spaces over the field $\mathbb{C}$, the almost paracomplex linear spaces are free modules over $\mathbb{A}$.

In a series of papers ([4]-[6]) M. Jurchescu defined and uses the notions of mixed linear space and mixed manifold having as (local) model the direct product $\mathbb{R}^{m} \times \mathbb{C}^{n}$. The mixed manifolds as differentiable families of complex spaces are useful at the cross-road of complex analysis and complex geometry, for example regarding the smoothly parameterized Čech cohomology of complex manifolds, [1]. Here, following his ideas and restricted at the linear level we define the concept of para-mixed linear space by replacing $\mathbb{C}$ with $\mathbb{A}$. A lot of properties of algebraic nature are similar to these frameworks and we hope to use further the notions considered now.

The paper is structured in two sections. The first one is devoted to the general theory of para-mixed linear spaces including the adapted linear maps between them and also their subspaces. A special attention is dedicated to the finite-dimensional case. The second section treats special morphisms between para-mixed linear spaces and the notion of paracomplexification of such spaces.

\section{Para-mixed linear spaces and subspaces}

Definition $1 A$ (almost) para-mixed linear space is a triple $\left(\mathrm{E}, \mathrm{E}_{\mathrm{p}}, \mathrm{P}\right)$ where $\mathrm{E}$ is a real linear space and $\mathrm{E}_{\mathrm{p}}$ is a linear subspace of $\mathrm{E}$ endowed with an (almost) paracomplex structure $\mathrm{P}$. Then $\mathrm{E}_{\mathrm{p}}$ is called the paracomplex part of $\mathrm{E}$ while the quotient real linear space $\mathrm{E}_{\mathrm{r}}=\mathrm{E} / \mathrm{E}_{\mathrm{p}}$ is the real part of $\mathrm{E}$. The para-mixed space is pure real if $\mathrm{E}_{\mathrm{p}}=0$ (i.e. $\mathrm{E}_{\mathrm{r}}=\mathrm{E}$ ) and pure paracomplex if $\mathrm{E}_{\mathrm{r}}=0$ (i.e. $\mathrm{E}_{\mathrm{p}}=\mathrm{E}$ ). In the following we place always in the "almost" case and for simplicity we will drop this epithet.

Example 1 i) The fundamental example is $\mathrm{E}=\mathrm{E}_{\mathrm{f}}=\mathbb{R}^{\mathrm{m}} \times \mathbb{A}^{\mathrm{n}}$ with $\mathrm{E}_{\mathrm{p}}=\mathbb{A}^{\mathrm{n}}$ and $\mathrm{E}_{\mathrm{r}}=\mathbb{R}^{\mathrm{m}}$. Hence, a para-mixed linear space can be thought as a (trivial) vector bundle over $\mathrm{E}_{\mathrm{r}}$ with paracomplex fibres $\mathrm{E}_{\mathrm{p}}$; also, para-mixed linear spaces can be though as linear families of paracomplex spaces. Let us point out that vertical bundles endowed with paracomplex structures are recently studied in [2] and the geometry of polynomial sub-endomorphisms on a pair of distributions for a given manifold are studied in [7].

ii) The paracomplex linear spaces will be considered as pure paracomplex para-mixed linear spaces while the real linear spaces will be considered as pure real para-mixed linear spaces. A para-mixed linear space $\mathrm{E}$ is simultaneous pure real and pure paracomplex if and only if $\mathrm{E}=\{0\}$. 
Definition 2 A linear map $\mathrm{T} \in \mathrm{L}_{\mathbb{R}}(\mathrm{E}, \mathrm{F}):=\mathrm{L}(\mathrm{E}, \mathrm{F})$ between two para-mixed linear spaces is called a morphism if $\mathrm{T}\left(\mathrm{E}_{\mathrm{p}}\right) \subseteq \mathrm{F}_{\mathrm{p}}$ and the induced map $\mathrm{T}_{\mathrm{p}}=$ $\left.\mathrm{T}\right|_{\mathrm{E}_{\mathrm{p}}}: \mathrm{E}_{\mathrm{p}} \rightarrow \mathrm{F}_{\mathrm{p}}$ is an $\mathbb{A}$-linear map. $\mathrm{T}$ is called an antimorphism if $\mathrm{T}\left(\mathrm{E}_{\mathrm{p}}\right) \subseteq \mathrm{F}_{\mathrm{p}}$ and $\mathrm{T}_{\mathrm{p}}$ is an $\mathbb{A}$-antilinear map: $\mathrm{T}(\mathrm{x}+\mathrm{jy})=\mathrm{T} \mathrm{x}-\mathrm{j} \mathrm{Ty} . \mathrm{T}_{\mathrm{p}}$ is the paracomplex part of $\mathrm{T}$ while $\mathrm{T}_{\mathrm{r}}=\left.\mathrm{T}\right|_{\mathrm{E}_{\mathrm{r}}}: \mathrm{E}_{\mathrm{r}} \rightarrow \mathrm{F}_{\mathrm{r}}$ is the real part of $\mathrm{T}$. Denotes by $\operatorname{Hom}(\mathrm{E}, \mathrm{F})$ the set of all morphisms from $\mathrm{E}$ to $\mathrm{F}$ and $\mathrm{T} \in \operatorname{Hom}(\mathrm{E}, \mathrm{F})$ is called isomorphism if both $\mathrm{T}_{\mathrm{p}}$ and $\mathrm{T}_{\mathrm{r}}$ are bijective maps.

Remark 1 i) If $\mathrm{E}$ is a pure real para-mixed linear space and $\mathrm{T} \in \mathrm{L}(\mathrm{E}, \mathrm{F})$ then $\mathrm{T}$ is both morphism and antimorphism. If $\mathrm{F}$ is a pure real para-mixed linear space and $\mathrm{T} \in \mathrm{L}(\mathrm{E}, \mathrm{F})$ then $\mathrm{T}$ is a morphism if and only if $\mathrm{T}_{\mathrm{p}}=0$. If $\mathrm{E}$ is a general para-mixed linear space then $\left(1_{\mathrm{E}_{r}}, \mathrm{P}\right) \in \operatorname{Hom}(\mathrm{E}, \mathrm{E})$ by considering the decomposition $\mathrm{E}=\mathrm{E}_{\mathrm{r}} \otimes \mathrm{E}_{\mathrm{p}}$ (see also the Corollary 1 below).

ii) The class of para-mixed linear spaces with their morphisms defines a category which contains the category of real linear spaces as well as the category of paracomplex linear spaces. The consideration of the paracomplex part (for spaces and morphisms) is a functor from the category of para-mixed linear spaces to the category of paracomplex linear spaces and similar for the consideration of the real part (for spaces and morphisms).

iii) Fix $\mathrm{E}$ and $\mathrm{F}$ two para-mixed linear spaces and $\mathrm{T}_{1}, \mathrm{~T}_{2} \in \mathrm{L}(\mathrm{E}, \mathrm{F})$ two (anti) morphisms. Consider also two real numbers $\alpha$, $\beta$. It follows that $\alpha \mathrm{T}_{1}+\beta \mathrm{T}_{2}$ is also an (anti) morphism with $\left(\alpha \mathrm{T}_{1}+\beta \mathrm{T}_{2}\right)_{i}=\left(\alpha \mathrm{T}_{1}\right)_{i}+\left(\beta \mathrm{T}_{2}\right)_{i}$ for $\mathrm{i} \in\{\mathrm{p}, \mathrm{r}\}$.

iv) The linear map $(x, y) \in \mathbb{R}^{2} \rightarrow z=x+j y \in \mathbb{A}$ is a bijective morphism which is not an isomorphism.

$\mathrm{v}) \operatorname{Hom}(\mathrm{E}, \mathrm{F})$ is a para-mixed linear space with the paracomplex part $\operatorname{Hom}(E, F)_{p}=\left\{T \in \operatorname{Hom}(E, F) ; T_{r}=0\right\}$ and real part $\operatorname{Hom}(E, F)_{r}=\operatorname{Hom}\left(E, F_{r}\right)$ $\simeq \operatorname{Hom}\left(\mathrm{E}_{\mathrm{r}}, \mathrm{F}_{\mathrm{r}}\right)$. The corresponding paracomplex structure is the map $\mathrm{T} \in \operatorname{Hom}(\mathrm{E}, \mathrm{F}) \rightarrow \mathrm{T} \circ\left(1_{\mathrm{E}_{\mathrm{r}}}, \mathrm{P}\right) \in \operatorname{Hom}(\mathrm{E}, \mathrm{F})$.

vi) Fix $\mathrm{T} \in \operatorname{Hom}(\mathrm{E}, \mathrm{F})$ and let $\mathrm{H}$ be another para-mixed linear space. Let $\mathrm{T}_{*}: \operatorname{Hom}(\mathrm{H}, \mathrm{E}) \rightarrow \operatorname{Hom}(\mathrm{H}, \mathrm{F})$ be the composition with $\mathrm{T}$ at left and $\mathrm{T}^{*}:$ $\operatorname{Hom}(\mathrm{F}, \mathrm{H}) \rightarrow \operatorname{Hom}(\mathrm{E}, \mathrm{H})$ be the composition with $\mathrm{T}$ at right. Then $\mathrm{T}_{*}$ and $\mathrm{T}^{*}$ are morphisms with respect to the para-mixed structure from $v$ ).

A first structural result is provided by:

Proposition 1 In the category of para-mixed linear spaces a given para-mixed linear space $\mathrm{E}$ is isomorphic with the direct product $\mathrm{E}_{\mathrm{r}} \times \mathrm{E}_{\mathrm{p}}$.

Proof. We have the canonical maps: $i: E_{p} \rightarrow E$ and $\pi: E \rightarrow E_{r}$. There exists the maps $q: E \rightarrow E_{p}$ and $\rho: E_{r} \rightarrow E$ such that: $q \circ i=1_{E_{p}}, i \circ q+\rho \circ \pi=1_{E}$. It 
follows that these maps $i, \pi, q, \rho$ are morphisms of para-mixed linear spaces and $(\rho, i): E_{r} \times E_{p} \rightarrow E$ is an isomorphism.

It follows directly:

Corollary 1 Let $\mathrm{E}=\mathrm{E}_{\mathrm{r}} \times \mathrm{E}_{\mathrm{p}}$ and $\mathrm{F}=\mathrm{F}_{\mathrm{r}} \times \mathrm{F}_{\mathrm{p}}$ be para-mixed linear spaces and $\mathrm{T} \in \mathrm{L}(\mathrm{E}, \mathrm{F})$. Then $\mathrm{T} \in \operatorname{Hom}(\mathrm{E}, \mathrm{F})$ if and only if it has the expression:

$$
\mathrm{T}=\left(\begin{array}{cc}
\mathrm{T}_{2} & 0 \\
\alpha & \mathrm{T}_{1}
\end{array}\right)
$$

where $\mathrm{T}_{1}: \mathrm{E}_{\mathrm{p}} \rightarrow \mathrm{F}_{\mathrm{p}}$ is a $\mathbb{A}$-linear map while $\mathrm{T}_{2}: \mathrm{E}_{\mathrm{r}} \rightarrow \mathrm{F}_{\mathrm{r}}$ and $\alpha: \mathrm{E}_{\mathrm{r}} \rightarrow \mathrm{F}_{\mathrm{p}}$ are real linear maps. In this decomposition, $\mathrm{T}_{1}$ is the paracomplex part of $\mathrm{T}$ and $\mathrm{T}_{2}$ is the real part of $\mathrm{T}$.

A characterization of isomorphisms is provided by:

Proposition 2 Let $\mathrm{E}, \mathrm{F}$ and $\mathrm{T}$ as above. Then the following statements are equivalent:

i) $\mathrm{T}$ is an isomorphism,

ii) $\mathrm{T}, \mathrm{T}_{1}$ and $\mathrm{T}_{2}$ are all bijective,

iii) two of the maps $\mathrm{T}, \mathrm{T}_{1}$ and $\mathrm{T}_{2}$ are bijective.

A special study can be performed in finite-dimension:

Definition 3 Let $\mathrm{E}$ be a para-mixed linear space and $\mathrm{m}, \mathrm{n} \in \mathbb{N}$. We say that $\mathrm{E}$ is of $(\mathrm{m}, \mathrm{n})$-type if $\mathrm{E}_{\mathrm{r}}$ is a real linear space of dimension $\mathrm{m}$ and $\mathrm{E}_{\mathrm{p}}$ is a paracomplex space of dimension $\mathrm{n}$. A frame on $\mathrm{E}$ is a set of vectors $\mathrm{B}=$ $\left\{e_{1}, \ldots, e_{\mathfrak{m}}, e_{\mathrm{m}+1}, \ldots, e_{\mathrm{m}+n}\right\}$ with $\left\{e_{\mathrm{m}+1}, \ldots, e_{\mathrm{m}+\mathrm{n}}\right\}$ a basis in $\mathrm{E}_{\mathrm{p}}$ and $\left\{\left[\mathrm{e}_{1}\right], \ldots,\left[\boldsymbol{e}_{\mathrm{m}}\right]\right\}$ a basis in $\mathrm{E}_{\mathrm{r}}$ where $[\mathrm{e}]$ is the class of $\mathrm{e} \in \mathrm{E}$ considered in $\mathrm{E}_{\mathrm{r}}$.

A characterization of this notion is:

Proposition 3 Fix $\mathrm{E}$ a para-mixed linear space of $(\mathrm{m}, \mathrm{n})$-type and $\mathrm{B}=\left\{\boldsymbol{e}_{1}, \ldots, \boldsymbol{e}_{\mathrm{m}+\mathrm{n}}\right\} \subset \mathrm{E}$ with $\left\{\mathrm{e}_{\mathrm{m}+1}, \ldots, \boldsymbol{e}_{\mathrm{m}+\mathrm{n}}\right\} \subset \mathrm{E}_{\mathrm{p}}$. Then $\mathrm{B}$ is a frame on $\mathrm{E}$ if and only if the map:

$$
\mathrm{T}:\left(x^{1}, \ldots, x^{\mathrm{m}+\mathrm{n}}\right) \in \mathrm{E}_{\mathrm{f}}=\mathbb{R}^{\mathrm{m}} \times \mathbb{A}^{\mathrm{n}} \rightarrow x=x^{i} e_{i} \in \mathrm{E}
$$

belongs to $\operatorname{Hom}\left(\mathrm{E}_{\mathrm{f}}, \mathrm{E}\right)$.

Definition 4 Let $\mathrm{E}$ be para-mixed linear space and $\mathrm{W}$ a subspace of $\mathrm{E}$. Then $\mathrm{W}$ is called para-mixed subspace of $\mathrm{E}$ if $\mathrm{W} \cap \mathrm{E}_{\mathrm{p}}$ is a paracomplex subspace of $\mathrm{E}_{\mathrm{p}}$ which means that $\mathrm{x} \in \mathrm{W} \cap \mathrm{E}_{\mathrm{p}}$ implies $\mathrm{jx} \in \mathrm{E}_{\mathrm{p}}$. 
A para-mixed subspace $W$ will be considered itself as a para-mixed linear space with $W_{p}=W \cap E$. Hence, the inclusion map $i: W \rightarrow E$ is a morphism with $i_{p}$ and $i_{r}$ injective maps.

Example 2 i) For $\mathrm{T} \in \operatorname{Hom}(\mathrm{E}, \mathrm{F})$ the kernel $\operatorname{ker} \mathrm{T}=\mathrm{T}^{-1}\left(0_{\mathrm{F}}\right)$ is a para-mixed subspace of $\mathrm{E}$.

ii) The intersection of an arbitrary family of para-mixed subspaces is again a para-mixed subspace.

iii) Let $\mathrm{E}$ be a pure real para-mixed linear space and $\mathrm{W} \subset \mathrm{E}$ a (real) subspace. Then $\mathrm{W}$ is a para-mixed subspace. A similar property holds for the paracomplex case.

\section{Monomorphisms and epimorphisms}

Definition 5 Let $\mathrm{T} \in \operatorname{Hom}(\mathrm{E}, \mathrm{F})$.

i) $\mathrm{T}$ is called monomorphism if there exists a para-mixed linear space $\mathrm{G}$ and $\mathrm{R} \in \operatorname{Hom}(\mathrm{G}, \mathrm{F})$ such that the map $(\mathrm{T}, \mathrm{R}): \mathrm{E} \times \mathrm{G} \rightarrow \mathrm{F}$ is an isomorphism.

ii) $\mathrm{T}$ is called epimorphism if there exists a para-mixed linear space $\mathrm{G}$ and $\mathrm{R} \in \operatorname{Hom}(\mathrm{E}, \mathrm{G})$ such that the map $(\mathrm{R}, \mathrm{T}): \mathrm{E} \rightarrow \mathrm{F} \times \mathrm{G}$ is an isomorphism.

A characterization of these types of morphisms is given by:

Proposition 4 Let $\mathrm{T} \in \operatorname{Hom}(\mathrm{E}, \mathrm{F})$ be given.

I) The following statements are equivalent:

a) $\mathrm{T}$ is a monomorphism,

b) $\mathrm{T}$ and $\mathrm{T}_{\mathrm{r}}$ are injective maps,

c) $T_{p}$ and $T_{r}$ are injective maps,

d) T have an inverse morphism at left.

II) Also, the following statements are equivalent:

e) $\mathrm{T}$ is an epimorphism,

f) $\mathrm{T}$ and $\mathrm{T}_{\mathrm{p}}$ are surjective maps,

g) $T_{p}$ and $T_{r}$ are surjective maps,

h) Thave an inverse morphism at right.

Proof. $a) \Rightarrow b)$. From hypothesis the maps $(T, R)$ and $(T, R)_{r}: E_{r} \times G_{r} \rightarrow F_{r}$ is bijective and then $\mathrm{T}, \mathrm{T}_{\mathrm{r}}$ are injective. $\left.\mathrm{b}\right) \Rightarrow \mathrm{c}$ ). It is obvious.

c) $\Rightarrow d$ ). Consider the decomposition (1) of $T$. Since $T_{p}$ is injective it follows the existence of $R_{1}$ an $\mathbb{A}$-linear map which is inverse at left. Similar, from $T_{r}$ being 
injective it result the existence of $R_{2}$ a $\mathbb{R}$-linear map which is inverse at left. The map $\mathrm{R}: \mathrm{F} \rightarrow \mathrm{E}$ given by:

$$
R=\left(\begin{array}{cc}
R_{r} & 0 \\
-R_{p} \alpha R_{r} & R_{p}
\end{array}\right)
$$

is a morphism from $F$ to $E$ with $R \circ T=I d_{E}$.

d) $\Rightarrow a)$. Let $R: F \rightarrow E$ be the inverse at left of $T$ and consider $G=\operatorname{ker} R$ together with the inclusion $i: G \rightarrow F$. From $R \circ\left(1_{F}-T \circ R\right)=0$ it results the existence of $w \in \operatorname{Hom}(F, G)$ such that $T \circ R+i \circ w=1_{F}$. Then $w \circ i=1_{G}$ and $w \circ T=0$. Let $A=(T, i): E \times G \rightarrow F$ and $B=(R, w): F \rightarrow E \times G$. With the equations above it follows that $A$ and $B$ are isomorphisms with $B=A^{-1}$. The equivalences from II are analogous.

Corollary 2 Fix $\mathrm{T} \in \operatorname{Hom}(\mathrm{E}, \mathrm{F})$. Then $\mathrm{T}$ is a monomorphism if and only if $\mathrm{T}(\mathrm{E})$ is a para-mixed subspace of $\mathrm{F}$ and the induced map $\mathrm{T}^{\prime}: \mathrm{E} \rightarrow \mathrm{T}(\mathrm{E})$ is an isomorphism. Also, $\mathrm{T}$ is an epimorphism if and only if the co-induced map $\mathrm{T}^{\prime \prime}: \mathrm{E} / \operatorname{ker} \mathrm{T} \rightarrow \mathrm{F}$ is an isomorphism.

Proof. Suppose that $T$ is a monomorphism. Since $T_{r}$ is injective it results that $T(E) \cap F_{p}=T_{p}\left(E_{p}\right)$ and so, $T(E)$ is a para-mixed subspace in $F$. It follows also that $T^{\prime} \in \operatorname{Hom}(E, T(E))$ and its paracomplex part $T_{p}^{\prime}$ is surjective. From $T=$ injective we get that $T^{\prime}$ and $T_{p}^{\prime}$ are bijective maps and then $T^{\prime}$ is an isomorphism. Similar arguments hold for the second part.

Let us remark that an injective $T \in \operatorname{Hom}(E, F)$ is not a-priori a monomorphism and the example is provided by the inclusion $\mathbb{R} \rightarrow \mathbb{A}$. In order to include this class we consider:

Definition $6 \mathrm{~T} \in \operatorname{Hom}(\mathrm{E}, \mathrm{F})$ is called cartesian monomorphism if it is injective and for every para-mixed linear space $\mathrm{G}$ and every map $\alpha: \mathrm{G} \rightarrow \mathrm{E}$ we have that $\mathrm{T} \circ \alpha$ is a morphism if and only if $\alpha$ is a morphism.

This notion is useful for another concept:

Definition 7 A paracomplexification of the para-mixed linear space $\mathrm{E}$ is a pair $\left(E^{p}, \rho\right)$ with $E^{p}$ a paracomplex linear space and $\rho \in \operatorname{Hom}\left(E, E^{p}\right)$ injective and satisfying $\rho(E)+j \rho(E)=E^{p}$ and $\rho(E) \cap j \rho(E)=\rho\left(E_{p}\right)$.

A characterization of this notion is given by: 
Theorem 1 i) Every para-mixed linear space E have a paracomplexification.

ii) The morphism $\rho$ is a cartesian monomorphism.

iii) A pair $\left(\mathrm{E}^{\mathfrak{p}}, \rho\right)$ is a paracomplexification of $\mathrm{E}$ if and only if $\mathrm{E}^{\mathfrak{p}}$ is a paracomplex linear space and the map $\rho^{*}: \operatorname{Hom}\left(\mathrm{E}^{p}, \mathrm{~F}\right) \rightarrow \operatorname{Hom}(\mathrm{E}, \mathrm{F})$ given in Remark 1(vi) is bijective for any paracomplex linear space $\mathrm{F}$.

Proof. i) Let $E=E_{r} \times E_{p}$ the canonical decomposition of $E$ and consider the space:

$$
E^{p}=\left(E_{r} \otimes_{\mathbb{R}} \mathbb{A}\right) \times E_{p} .
$$

It results that $E^{p}$ is a paracomplex linear space. One define the map $\rho: E \rightarrow E^{p}$ by $\rho\left(x_{2}, x_{1}\right)=\left(x_{2} \otimes 1, x_{1}\right)$ and a straightforward computation gives that $\left(E^{p}, \rho\right)$ is a paracomplexification of $E$.

ii) Let $G$ be a para-mixed linear space and $\alpha: G \rightarrow E$ such that $\rho \circ \alpha \in$ $\operatorname{Hom}\left(G, E^{p}\right)$. Fix $z \in G_{p}$; then:

$$
\rho \circ \alpha(z)=j(\rho \circ \alpha)(j z) \in \rho(E) \cap j \rho(E)=\rho\left(E_{p}\right)
$$

and the injectivity of $\rho$ yields that $\alpha(z) \in \mathrm{E}_{\mathrm{p}}$. Also, $\rho \circ \alpha(j z)=j(\rho \circ \alpha(z))=$ $\rho(j \alpha(z))$ and again the injectivity of $\rho$ gives $\alpha(j z)=j \alpha(z)$. These facts together with the $\mathbb{R}$-linearity means that $\alpha \in \operatorname{Hom}(G, E)$.

iii) Fix $\left(E^{p}, \rho\right)$ a paracomplexification of $E, F$ a paracomplex linear space and $u \in \operatorname{Hom}(E, F)$. Define then $v: E^{p} \rightarrow F$ :

$$
v(\rho(x)+j \rho(y)):=u(x)+j u(y)
$$

for all $x, y \in E$. Since $\rho(x)+j \rho(y)=\rho\left(x^{\prime}\right)+j \rho\left(y^{\prime}\right)$ if and only if $y^{\prime}-y \in E_{p}$ and $x-x^{\prime}=j\left(y^{\prime}-y\right)$ it results that $v$ is well defined. It follows that $v$ is a $\mathbb{A}$-linear map and $v \circ \rho=u$. We get also the uniqueness of $v$ with these two properties.

Example 3 i) Let $\mathrm{E}$ be a real linear space endowed with the paracomplex structure $\mathrm{J}$. Consider then $\mathrm{E}^{p}=(\mathrm{E}, \mathrm{J}) \oplus(\mathrm{E},-\mathrm{J})$ and the diagonal map $\rho: \mathrm{E} \rightarrow \mathrm{E}^{p}$. Then $\left(\mathrm{E}^{\mathrm{p}}, \rho\right)$ is a paracomplexification of $\mathrm{E}$. Indeed, every vector $\left(\mathrm{e}_{1}, \mathrm{e}_{2}\right) \in \mathrm{E}^{\mathrm{p}}$ has a decomposition $\left(\mathrm{e}_{1}, \mathrm{e}_{2}\right)=(\mathrm{x}+\mathrm{Jy}, \mathrm{x}-\mathrm{Jy})$ with $\mathrm{x}, \mathrm{y} \in \mathrm{E}$. More precisely, $x=\frac{1}{2}\left(e_{1}+e_{2}\right)$ and $y=\frac{1}{2}\left(\mathrm{Je}_{1}-\mathrm{Je}_{2}\right)$.

Let now $\mathrm{F}$ be a paracomplex linear space and $\mathrm{u}: \mathrm{E} \rightarrow \mathrm{F}$ a $\mathbb{R}$-linear map. The unique $\mathbb{A}$-linear map $v: \mathbb{E}^{p} \rightarrow F$ satisfying $v \circ \rho=u$ is:

$$
v\left(e_{1}, e_{2}\right)=\frac{1}{2}\left(u\left(e_{1}\right)-j u\left(J e_{1}\right)\right)+\frac{1}{2}\left(u\left(e_{2}\right)+j u\left(J e_{2}\right)\right) .
$$


ii) Let $\mathrm{E}, \mathrm{F}$ be para-mixed linear spaces and fix $\rho: \mathrm{F} \rightarrow \mathrm{F}^{\mathrm{p}}$ a paracomplexification of $\mathrm{F}$. Then $\rho_{*}: \operatorname{Hom}(\mathrm{E}, \mathrm{F}) \rightarrow \operatorname{Hom}\left(\mathrm{E}, \mathrm{F}^{\mathrm{p}}\right)$ given in Remark 1(vi) is a cartesian monomorphism. In general, $\left(\operatorname{Hom}\left(\mathrm{E}, \mathrm{F}^{\mathrm{p}}\right), \rho_{*}\right)$ is not a paracomplexification of $\operatorname{Hom}(\mathrm{E}, \mathrm{F})$ since $\operatorname{Hom}\left(\mathrm{E}, \mathrm{F}^{\mathrm{p}}\right)$ is a paracomplex linear space satisfying $\rho_{*}\left(\operatorname{Hom}\left(E, F^{p}\right)\right) \cap j \rho_{*}\left(\operatorname{Hom}\left(E, F^{p}\right)\right)$ but generally it do not satisfies $\rho_{*}\left(\operatorname{Hom}\left(E, F^{p}\right)\right)+j \rho_{*}\left(\operatorname{Hom}\left(E, F^{p}\right)\right)=\left(\rho_{*}\left(\operatorname{Hom}\left(E, F^{p}\right)\right)\right)^{p}$.

\section{References}

[1] T. Bailey, M. Eastwood, S. Gindikin, Smoothly parameterized Čech cohomology of complex manifolds, J. Geom. Anal., 15 (2005), no. 1, 9-23. MR2132263 (2006b:32013)

[2] M. Crasmareanu, L.-I. Pişcoran, Weak para-CR structures on vertical bundles, Adv. Appl. Clifford Algebr., 26 (2016), no. 4, 1127-1136. MR3564462

[3] V. Cruceanu, P. Fortuny; P. M. Gadea, A survey on paracomplex geometry, Rocky Mountain J. Math., 26 (1996), no. 1, 83-115. MR1386154 (97c:53112)

[4] M. Jurchescu, Variétés mixtes, in Romanian-Finnish Seminar on Complex Analysis (Proc., Bucharest, 1976), 431-448, Lecture Notes in Math., 743, Springer, Berlin, 1979. MR0552907 (80m:32011)

[5] M. Jurchescu, Espaces mixtes, in "Complex analysis"-Fifth RomanianFinnish Seminar, Part 2 (Bucharest, 1981), 37-57, Lecture Notes in Math., 1014, Springer, Berlin, 1983. MR0738113 (85j:32048)

[6] M. Jurchescu, Variétés mixtes et cohomologie, Riv. Mat. Univ. Parma, (4) 10 (1984), special vol. 10, 55-79. MR0777315 (86e:32036)

[7] F. Özdemir; M. Crasmareanu, Geometrical objects associated to a substructure, Turkish J. Math., 35 (2011), no. 4, 717-728. MR2896417 